\title{
Análisis de la Logística Inversa de Productos Perecederos en México
}

\author{
Analysis of Reverse Logistics of Perishable Products in Mexico \\ Evangelina Lezama-León ${ }^{a}$, Myrna H. Lezama-León ${ }^{b}$, Alonso E. Solís-Galindo ${ }^{c}$, Héctor \\ Figueroa-Urrea $^{d}$
}

\begin{abstract}
:
Population growth involves certain social problems, one of them is the increase in the demand for perishable products. Attent ion to one of the basic needs such as food becomes unsustainable in most developing countries (Mexico, Central and South American countries, among others), since statistically the population grows faster than the production of resources food available. One of the strategies being carried out by the food industry is to optimize the use of food, avoiding waste due to expiration time or prepared surplus. In this research, an exploratory study is presented through a review of the current literature in databases and publications that present the state of the art on this topic. The result of the investigation are the studies on the techniques of the Reverse Logistics (LI) process applied to the recovery of perishable products in Mexico, specifically food, presenting a classification table on the types of waste.
\end{abstract}

Keywords:

Reverse logistics, perishable products, strategy

\section{Resumen:}

El crecimiento demográfico conlleva ciertos problemas sociales, uno de ellos es el aumento en la demanda de productos pereced eros. La atención a una de las necesidades básicas como la alimentación se torna insostenible en la mayoría de los países en vías de desarrollo (México, países de centro y Sudamérica, entre otros), ya que estadísticamente la población crece más rápido que la producción de recursos alimenticios disponibles. Una de las estrategias que se está llevando a cabo por la industria alimenticia es optimizar el uso de los alimentos evitando el desperdicio por tiempo de caducidad o por excedente preparado. En esta investig ación se presenta un estudio exploratorio a través de la revisión de la literatura actual en bases de datos y publicaciones que presentan el estado del arte en este tema. El resultado de la investigación son los estudios sobre las técnicas del proceso de Logística Inversa (LI) aplicadas a la recuperación de los productos perecederos en México, específicamente alimentos, presentando una tabla de clasificación sobre los tipos de residuos.

\section{Palabras Clave:}

Logística inversa, productos perecederos, estrategia

\section{Introducción}

En el mundo, existen aproximadamente 1.3 billones de toneladas de desperdicio o pérdida de alimentos. En los países ricos el desperdicio es de 222 millones de toneladas de la producción total aproximadamente (Save food.org). En México, se desperdician 20.4 millones de toneladas de alimentos cada año, lo que representa impactos ambientales por el uso inapropiado de agua y energía, dicho estudio se basa en una muestra de 79 alimentos estudiados, así mismo este publicación indica que se están generando además 36 millones de toneladas de dióxido de carbono ( $\mathrm{CO} 2$ ), el equivalente a lo que generan 15.7 millones de vehículos anualmente , por otra parte, en la producción de alimentos que se desperdician, se pierden más de 40 billones de litros de

\footnotetext{
a Universidad Autónoma del Estado de Hidalgo, https://orcid.org/0000-0003-0818-0897, Email: evangeli@uaeh.edu.mx

b Universidad Politécnica Metropolitana de Hidalgo, https://orcid.org/0000-0001-8181-3705, Email: mlezama@ upmh.edu.mx

c Universidad Autónoma del Estado de Hidalgo, https://orcid.org/0000-0002-3999-006X, Email: soliser@uaeh.edu.mx

d Universidad Politécnica Metropolitana de Hidalgo, https://orcid.org/0000-0001-6240-0470, Email: hfigueroa@upmh.edu.mx
} 
agua , que se puede comparar con la cantidad utilizada por los mexicanos en 2.4 años [1]. De acuerdo con un estudio del Banco Mundial, sería posible atender la deficiencia en cuanto a alimentación de 7,4 millones de mexicanos o más con solo recuperar los alimentos que se desperdician. En México se importa entre un 20 y un $50 \%$ consume, que podría parecer contradictorio con la cantidad de comida desperdiciada [2], ver figura 1.

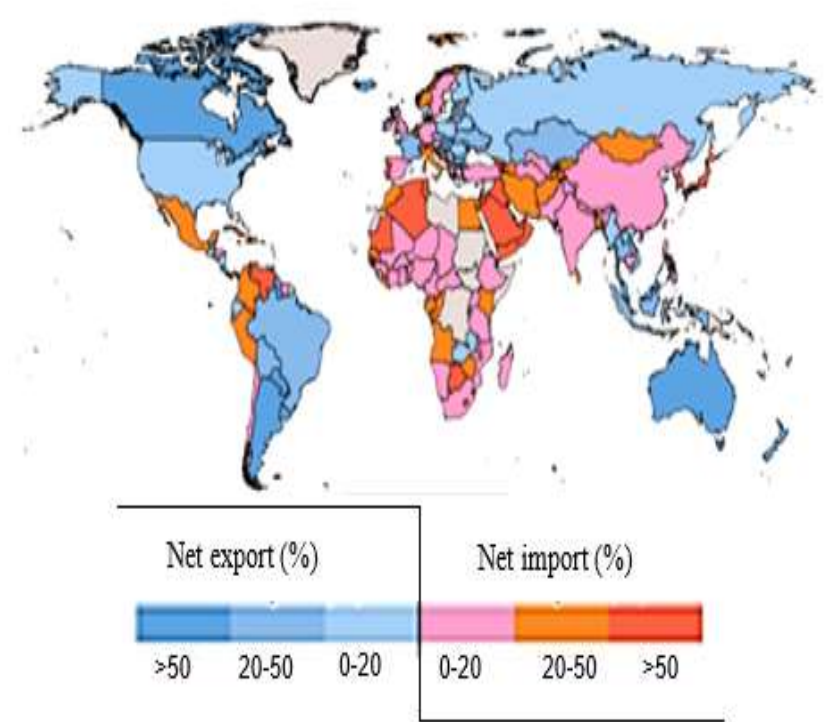

Figura 1. Importación de alimentos [3].

El objetivo de este trabajo es conocer y explicar los avances que se tienen hasta el momento en el manejo de residuos en cuanto a la logística inversa y conocer cómo se está llevando a cabo el manejo de residuos de alimentos para su reutilización en la industria, específicamente en restaurantes. Se describe por lo tan to el concepto de logística inversa, los trabajos actuales que muestran el avance en este tema y el manejo que se le da a los residuos, con un enfoque en los alimentos en la industria en México.

\section{Una aproximación al estudio del manejo de los desechos en los productos perecederos}

Los productos que son perecederos tienen un tiempo de vida limitado, generalmente se ubican dentro de los productos perecederos los alimentos, flores, medicinas, incluso los diarios impresos debido a que conservan su valor sólo hasta determinado tiempo perdiendo su valor después del día que salen a la venta. En este trabajo, únicamente se estudiarán los alimentos.

Los productos perecederos son elementos con una vida útil corta medido en días contados a partir del día en que se producen hasta que este se vuelve inaceptable para su consumo.
En [4], artículo sobre control de inventario de perecederos en supermercados se definen como perecederos a elementos que tienen una vida útil menor o igual a 30 días, esto al determinar el umbral para el tiempo de conservación. En su estudio, el examen de los datos reveló que todos los productos que requieren un ambiente acondicionado tienen una vida útil de 30 días.

Existen factores en la cadena de suministro de productos agroalimentarios (perecederos), como son calidad de los productos, la segu ridad agroalimentaria, la variabilidad de la demanda, uso de transporte eficiente, tiempo de transporte y los costos de entrega, la disponibilidad de los productos, distancia que debe recorrer el producto, la presencia de fenómenos naturales, trazabilidad 0 localización, entre otros, de manera que la suma de todos estos factores hacen que la gestión de cadenas de suministro de productos agroalimentarios difiera con otras [5], sin embargo, el factor tiempo es el más significativo en la complejidad para su manejo.

Las pérdidas de alimentos se dan desde la fase de producción, la postcosecha y el procesamiento en la cadena de suministro [6]. Al final de la cadena alimentaria, a las pérdidas se le llaman desechos de alimentos, lo cual tiene que ver con el comportamiento del consumidor final [6]. En la literatura se enfatiza la diferencia entre los términos, pérdida de alimento (Food looses) y el desecho de alimentos (Food waste). El primero se refiere a los alimentos que son rechazados por un mal manejo de ellos dentro de la cadena de suministro y los desechos son los que así se consideran porque han llegado a una fecha de caducidad o por un excedente de preparación, es decir, no son consumidos porque la oferta excede la demanda.

Los alimentos al no ser destinados para lo que fueron planificados, a pesar de que puedan ser utilizados, por ejemplo, para generar bioenergía, se siguen considerando como pérdidas.

A nivel mundial, millones de alimentos terminan en la basura o se malogran en el transporte a los mercados, por lo que debe enfrentarse el problema en cada país con el fin de lograr la seguridad en alimentación y terminar con la pobreza [7].

Existen iniciativas para solventar el problema del desperdicio de alimentos, sin embargo, se sigue desperdiciando un tercio de la producción de alimentos a nivel mundial, se ha prestado poca atención a este problema y no se han realizado estudios exhaustivos que evalúen la magnitud del problema que esto representa en el medio ambiente [8].

A continuación, se muestran los porcentajes de residuos en la cadena alimentaria por tipo de alimento. 


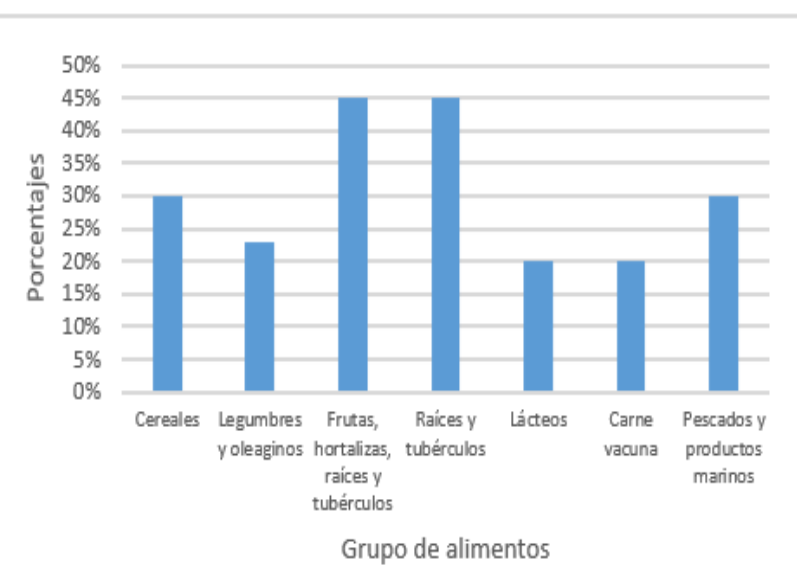

Figura 2. El porcentaje de residuos en la cadena alimentaria [8].

A pesar de que la disponibilidad energética para México es de los más elevados en el mundo, existe malnutrición, esto es, desnutrición y obesidad. Un $18 \%$ de la población está en pobreza alimentaria por ingreso, concentrándose en siete estados de la República Mexicana [9].

Tanto los desechos como la pérdida de alimentos contribuyen en la emisión de gases de efecto invernadero al llegar a los vertederos, así como en las actividades de producción, procesamiento, transporte, almacenamiento y distribución. Dentro de los impactos ambientales se encuentran el agotamiento de los recursos naturales, en cuanto al suelo, los nutrientes, el agua y energía entre otros impactos, además, desde el punto de vista social, en las dimensiones ética y social en el concepto de seguridad global [10].

\subsection{Manejo de desechos de perecederos en México}

En México existen programas de manejo de residuos sólidos urbanos que incluyen todos los desechos, tal es el caso de los desperdicios de alimentos que se generan en hogares o la industria en general, así como residuos de manejo especial. Algunos estados de la república cuentan con un programa para la prevención y gestión integral de residuos sólidos. De manera general los datos a nivel nacional que se encuentran registrados hasta el momento en las bases de datos de INEGI se muestran en la tabla 1.

\begin{tabular}{|l|l|r|c|}
\hline \multicolumn{1}{|c|}{ Descripción } & Unidad & Cantidad & Periodo \\
\hline $\begin{array}{l}\text { Promedio diario de } \\
\text { residuos sólidos urbanos } \\
\text { recolectados a nivel } \\
\text { nacional }\end{array}$ & Kilogramos & $102,887,315$ & 2014 \\
\hline $\begin{array}{l}\text { Número de vehículos } \\
\text { utilizados para } \\
\text { recolección de residuos } \\
\text { sólidos urbanos a nivel } \\
\text { nacional con } \\
\text { compactador }\end{array}$ & Número & 10,187 & 2014 \\
\hline $\begin{array}{l}\text { Cantidad promedio } \\
\text { diaria de residuos }\end{array}$ & Kilogramos & $2,748,727$ & 2012 \\
\hline
\end{tabular}

\begin{tabular}{|l|l|r|r|}
\hline $\begin{array}{l}\text { orgánicos enviados a } \\
\text { tratamiento }\end{array}$ & & \\
\hline $\begin{array}{l}\text { Cantidad de residuos } \\
\text { inorgánicos enviados a } \\
\text { tratamiento }\end{array}$ & Kilogramos & $6,083,199$ & 2012 \\
\hline
\end{tabular}

Tabla1. Manejo de residuos sólidos urbanos [11].

En la ciudad de México y zona conurbada, existen más de 20 millones de personas que generan aproximadamente entre 1 y 1.5 kilos de residuos sólidos, reciclándose únicamente el $18 \%$ de los desechos en los hogares, como es el caso de la ciudad de México, donde existe la Ley General para la Prevención y Gestión Integral de los Residuos, publicada en el Diario Oficial desde el año 2003. Este tipo de leyes tienen más éxito en países como Alemania o Suecia donde el porcentaje de residuos que llega a los rellenos sanitarios es del $4 \%$, comparándolo con México que asciende a un 70\% [12].

En el manejo de residuos se generan gastos para unos y ganancias para otros. En el caso de la ciudad de México existen 14 mil 140 trabajadores pagados por el gobiemo de dicha ciudad, entre sindicalizados y eventuales, además de los voluntarios que son aproximadamente 10 mil. Estos trabajadores separan lo que es de valor y lo venden por su cuenta, lo que les permite generar un ingreso extra, entre los que se encuentran el cartón, papel, metal y vidrio. Una vez separados los desechos, el resto es llevado a los rellenos sanitarios.

El uso de desechos alimenticios puede generar biocombustibles, como el biocarbon que puede ser generado a escala comercial en México o los biocombustibles principales que se muestran en la tabla 2. El aprovechamiento de los desechos de alimentos para la generación de bioenergía ha sido de interés para investigadores y asociaciones como la red Mexicana de Bioenergía entre otras.

\begin{tabular}{|l|l|l|l|}
\hline Bioenergéticos & Sólidos & Líquidos & Gaseosos \\
\hline $1^{\underline{a}}$ generación & $\begin{array}{l}\text { Leña, } \\
\text { carbón } \\
\text { vegetal, } \\
\text { bagazo, } \\
\text { pellets }\end{array}$ & $\begin{array}{l}\text { Bioetanol, } \\
\text { biodiesel, } \\
\text { licor negro }\end{array}$ & $\begin{array}{l}\text { Biogás, gas } \\
\text { de síntesis }\end{array}$ \\
\hline $2^{\text {a }}$ generación & $\begin{array}{l}\text { Biochar, } \\
\text { torrefactos, } \\
\text { torpellets }\end{array}$ & $\begin{array}{l}\text { Etanol } \\
\text { celulósico, } \\
\text { syndiésel, } \\
\text { aceite de } \\
\text { pirolisis. }\end{array}$ & Biometano \\
\hline $3^{\text {a }}$ generación & & $\begin{array}{l}\text { Diésel de } \\
\text { algas, etanol } \\
\text { de algas }\end{array}$ & Biohidrógeno \\
\hline
\end{tabular}

Tabla 2. Principales biocombustibles [13].

En México la bioenergía representa un 4.2\% del total, donde las tecnologías para el aprovechamiento energético de la biomasa en su mayoría son ineficientes e incluso pueden tener efectos negativos para la salud y el medio ambiente [13]. 


\section{El estudio exploratorio como un tipo de herramienta para la explicación del uso de los residuos perecederos.}

El problema que se presenta en este trabajo requiere un estudio exploratorio como un tipo de metodología, para explicar el manejo de los desechos perecederos.

En cuanto a los enfoques que han abordado los estudios de corte exploratorio en logística inversa se ha encontrado la revisión del problema de ruteo de vehículos, es decir, desde el punto de vista de la optimización del transporte por medio de diversas técnicas como son las heurísticas, así como diversos modelos de solución al problema.

Dentro de este enfoque uno de los objetivos es la optimización de costos en transportación.

Dentro de los enfoques relacionados a la logística de productos perecederos, en específico el caso de los alimentos, se encuentran el enfoque de la estrategia de clusters, el enfoque del reciclaje para bancos de alimentos, el aprovechamiento y uso industrial de los desperdicios de alimentos destinados al consumo humano, y la cadena de suministros.

Una vez que se ha obtenido la información se desarrolla una clasificación portipo de producto, tomando en cuenta únicamente productos perecederos, considerando el enfoque desde el que se está abordando y la referencia que nos da más información del lugar donde se realizó el estudio citado.

Se considera en primer lugar los estudios aplicados en México, sin embargo se han incluido algunos estudios de otros países para tener una referencia y de esta manera analizar las áreas de oportunidad en el país.

\section{Un análisis a los procesos de recuperación de residuos}

En este apartado, se explica el proceso de recuperación de residuos, a través, de lo que llamamos Logística Inversa (L.I.).

La Logística es una actividad empresarial que se aplica a las funciones de suministro, almacenamiento, producción, distribución y consumo y se divide en Logística de flujo directo (hacia adelante), y logística de flujo inverso (hacia atrás) como se muestra en la figura 3. La logística inversa o de flujo inverso se refiere a la recuperación de valor de los productos que van del consumidor a la planta de producción para su aprovechamiento. La logística de flujo inverso o logística inversa tiene actividades como la recolección, clasificación/inspección, reproceso y redistribución. El ejemplo más claro es en el reciclaje de botellas pet, que son regresadas para su tratamiento y generación de nuevas botellas.
En el estudio [15] sobre el impacto de la logística inversa en la disposición de plástico y cartón en tres supermercados de Sao Paulo, Brasil durante seis meses, arrojó como resultado una reducción de 220 toneladas de materiales abióticos y bióticos, así como la disminución en disminución en la contaminación de agua y aire.

La logística inversa, que trata con los flujos físicos de productos, componentes y materiales que fluyen de usuarios / propietarios a reutilizadores, es un campo en crecimiento tanto en la práctica como en el mundo académico, además, con el fin de obtener una idea de cómo se maneja la logística inversa en la práctica, los estudios de campo y las encuestas son útiles, así mismo, en su estudio se consideran casos de logística inversa entre los que se encuentra el manejo de productos como baterías, cartuchos de toner, botellas pet, papel, entre otros [16].

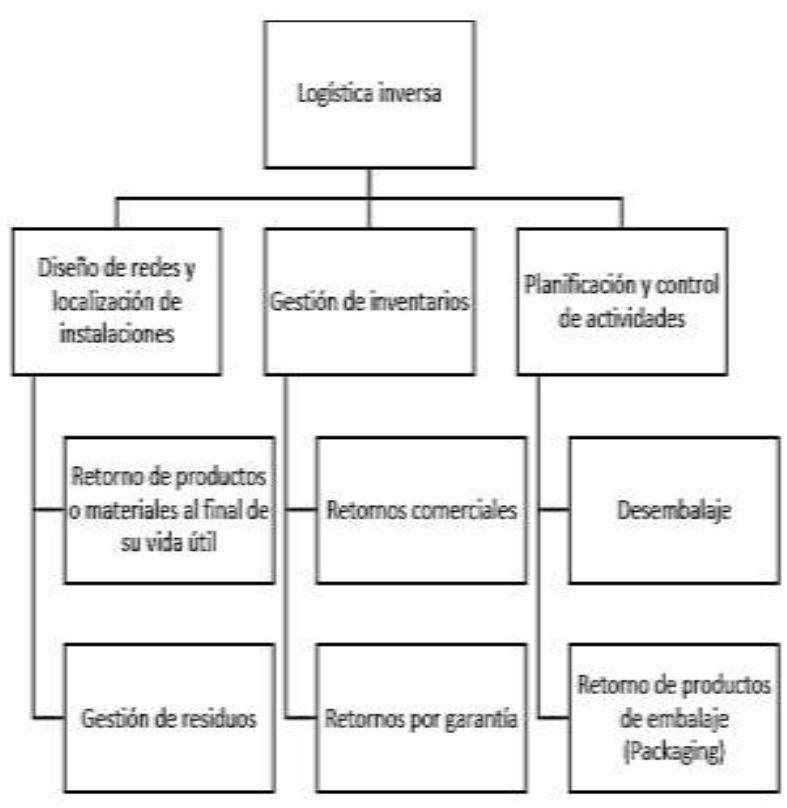

Figura 3. Problemas abordados desde la logística inversa [14].

\subsection{La aplicación de la Logística Inversa en residuos de alimentos}

Existen estudios realizados para atacar el problema de logística inversa de transporte de residuos alimenticios, como el desarrollado en [17], donde se analiza la reducción de la distancia total de la ruta de la logística inversa, con respecto al peso total del desperdicio de alimentos en cada área de recolección aplicando un modelo CVPR (Problema de enrutamiento de vehículos capacitados), dicho estudio se realizó en la República de Corea, para definir las rutas diarias óptimas de acuerdo con la cantidad de residuos en cada área de recolección, los cuales serán indicados utilizando tecnología RFID, comparando los resultados obtenidos con el método de 
barrido (para representar la situación actual), con el método CVRP fijo y finalmente con el método dinámico diario CVRP, en donde, el mejor resultado fue este último logrando reducir la distancia total recorrida en un promedio del $30.76 \%$ con respecto al método de barrido en un $10.13 \%$ con respecto el método CVRP fijo.

Por su parte en el artículo CVRPTW model applied to the collection of food donations, realizaron un modelo matemático de del tipo CVRPTW (Problema Capacitado de Ruteo de Vehículos con Ventanas de Tiempo para minimizar el costo de transporte de las rutas de recolección de las donaciones de un banco de alimentos en la ciudad de Bogotá, Colombia, se resolvió con programación lineal entera mixta, intentan do estandarizar dicho modelo para la solución de este tipo de casos [18]. Así mismo en [19], realizaron el estudio de un sistema de logística inversa, con un modelo de multiperiodo y multiescalonamiento, para su solución se aplicaron los algoritmos de Sistema Inmunitario Artificial (AIS) y la Optimización de Enjambre de Partículas (PSO), obteniendo mejores resultados con el primero, es decir, maximizando las ganancias esperadas.

\subsection{Manejo de residuos de alimentos}

En otros países, se ha desarrollado investigación para el aprovechamiento de residuos de cocinas donde se analiza el manejo del desperdicio de aceite de cocina en China y Japón. Los resultados de [20] muestran que los subsidios que se proporciona a los recicladores en estos países no son significativos, a diferencia de las empresas dedicadas a la producción de Biocombustible donde este apoyo representa una mejoría significativa en las tasas de recuperación. En [20] se afirma que la mejora a la infraestructura de reciclaje, la implementación de un estricto control técnico y administrativo y la política de ventas creativas de productos acabados de biocombustibles pueden beneficiar la tasa de recuperación en China.

En cuanto al tratamiento de desechos orgánicos, la investigación de [21] Adi y Noor (2009) muestra que el aprovechamiento de desechos de cocina en combinación con estiércol de vaca, en específico los granos de café de desperdicio, mostró un mayor porcentaje de elementos nutritivos para la producción de vermicomposta.

En [22] se afirma que las soluciones más demandadas en cuanto a desechos de alimentos se refieren es tanto evitar el desperdicio como donar fracciones comestibles para servicios sociales. Estos autores revisan el estado del arte en cuanto al aprovechamiento y uso industrial de los desperdicios de alimentos destinados al consumo humano, resultando estrategias y tecnologías de conversión, incluyendo el surgimiento del concepto de biorrefinería al generar biocombustible con estos desechos.

En [23] se modelan tres partes, incluyendo el gobierno, las empresas de biocombustible y restaurantes bajo el supuesto de que existe falta de información en la cadena de suministro de aceite de cocina de desecho, proponiendo que las empresas de biocombustible además de los restaurantes, sean una parte activa en la logística inversa, donde el gobierno debería eliminar las tarifas de disposición de basura que se aplican a los restaurantes y aumentar los subsidios, mencionando que los restaurantes deben ser el centro de atención en este tema.

Los principales aliados en el manejo de los desperdicios en México, son los bancos de alimentos, por ejemplo APT (Alimentos para todos) que opera desde hace 23 años, principalmente en la central de abastos, empresas de la industria alimentaria y supermercados recuperando aproximadamente 900 toneladas mensuales de comida para beneficiar a $60 \mathrm{mil}$ personas rescatando los alimentos que aún son aptos para consumo humano sin poner en riesgo la salud, como son, productos de caducidad próxima a vencer, o productos que estén maltratados y que no es atractivo o haya perdido su valor comercial [24].

Se creó la Red Temática en Seguridad Alimentaria: Valorización de Residuos Agroindustriales y Disminución de Pérdidas y Desperdicios de Alimentos, también conocida como Red PDA (Pérdida y Desperdicio de Alimentos). Esta red es una iniciativa de investigadores mexicanos multidisciplinaria con el fin de evitar el desperdicio de alimentos y propicia la seguridad alimentaria a través de la concientización y la investigación [25]. Se resume en la tabla 3 los trabajos sobre el manejo de residuos de alimentos.

\section{Conclusiones}

Existe un área de oportunidad importante en la optimización del consumo de los alimentos producidos en México, en primer lugar, para satisfacer la necesidad de alimentación en el país al $100 \%$ y en segundo lugar, reducir los niveles de consumo de energía y recursos así como las importaciones.

La mayor parte del desperdicio de alimentos se genera en la cadena de abastecimiento (food loss) y en menor porcentaje en el usuario final. A pesar de ello, se genera una importante cantidad de residuos que pueden ser aprovechados. Los desperdicios que se generan por el usuario (food waste) se presentan cuando los alimentos cumplen la fecha de caducidad o debido al excedente de producción. Existe investigación actual enfocada en la 
solución del problema en la logística de flujo directo y estudios en la logística inversa, en su mayoría, estos son enfocados a residuos. Los estudios formales de logística inversa de alimentos para su aprovechamiento y recuperación de valor se relacionan con el manejo de los residuos como el aceite para generarbiocombustible y los residuos orgánicos para la generación de biogás. Las investigaciones mencionadas e este trabajo contemplan la optimización de rutas de vehículos con el fin de reducir el impacto por la generación de contaminación, con diversos métodos como son las heurísticas. En cuanto a la recuperación de alimentos para consumo humano se encuentran propuestas en investigaciones formales referentes a la logística inversa en otros países. No se encontraron estudios sobre el manejo o logística inversa de alimentos en México, existien do muy poca información al respecto, a pesar de ello, se emprenden acciones a través bancos de alimentos y de voluntarios para la recuperación de alimentos para consumo humano provenientes de centrales de abastos o supermercados. La aplicación de estrategias en el manejo adecuado y aprovechamiento de desperdicios de alimentos es un área de oportunidad en México, principalmente, por el impacto en ahorro para las empresas y en el abastecimiento de alimentos para la población, incluyendo el sector más vulnerable que puede aprovechar los alimentos desechados. Desde este enfoque se propone como trabajo futuro la generación de un mapa de ruta por entidad en el tema del manejo y aprovechamiento de alimentos para el consumo humano y generación de bioenergía, que permitirán instrumentar y comunicar la estrategia centrándose en la innovación para el desarrollo sostenible y sustentable.

\begin{tabular}{|c|c|c|}
\hline $\begin{array}{l}\text { Tipo de } \\
\text { residuo }\end{array}$ & $\begin{array}{c}\text { Descripción del } \\
\text { manejo o propuesta }\end{array}$ & Referencia \\
\hline $\begin{array}{l}\text { Aceite } \\
\text { comestible }\end{array}$ & $\begin{array}{l}\text { Uso como } \\
\text { biocombustible } \\
\text { Concepto de } \\
\text { biorrefinería } \\
\text { Las empresas de } \\
\text { biocombustible además } \\
\text { de los restaurantes, } \\
\text { sean una parte activa } \\
\text { en la logística inversa, } \\
\text { donde el gobierno } \\
\text { debería eliminar las } \\
\text { tarifas de disposición de } \\
\text { basura que se aplican a } \\
\text { los restaurantes y } \\
\text { aumentar los subsidios }\end{array}$ & $\begin{array}{l}\text { Zhang, et. al } \\
\text { (2015) } \\
\text { Girotto, } \\
\text { Alibardi y } \\
\text { Cossu, } \\
\text { (2015) } \\
\text { Zhang, Xu, } \\
\text { Zhou y Cao } \\
\text { (2017) }\end{array}$ \\
\hline $\begin{array}{l}\text { Alimentos } \\
\text { recuperados } \\
\text { de central de } \\
\text { abastos, } \\
\text { empresas de } \\
\text { la industria } \\
\text { alimentaria o } \\
\text { supermercad } \\
\text { os }\end{array}$ & $\begin{array}{l}\text { Recuperación de } \\
\text { alimentos para } \\
\text { consumo humano a } \\
\text { través de banco de } \\
\text { alimentos }\end{array}$ & $\begin{array}{l}\text { Medina } \\
(2017)\end{array}$ \\
\hline $\begin{array}{l}\text { Alimentos en } \\
\text { general }\end{array}$ & $\begin{array}{l}\text { Evitar la pérdida de } \\
\text { alimentos a través de la } \\
\text { concientización y la } \\
\text { investigación } \\
\text { Sugiere la reducción de } \\
\text { la distancia total de la } \\
\text { ruta de la logística } \\
\text { inversa. }\end{array}$ & $\begin{array}{l}\text { Medina } \\
\text { (2016) } \\
\text { Kim, Kang y } \\
\text { Kim (2014) }\end{array}$ \\
\hline $\begin{array}{l}\text { Desechos de } \\
\text { cocina, en } \\
\text { especial } \\
\text { granos de } \\
\text { café }\end{array}$ & $\begin{array}{l}\text { Combinación de } \\
\text { desechos combinado } \\
\text { con estiércol para } \\
\text { producción de } \\
\text { vermicomposta }\end{array}$ & $\begin{array}{l}\text { Adi y Noor } \\
(2009)\end{array}$ \\
\hline
\end{tabular}

Tabla 3. Resumen del manejo de residuos de alimentos

\section{Referencias}

[1] SEMARNAT. (7 de diciembre de 2017). Comunicado de Prensa Núm. 123/17. Recuperado el 04 de 05 de 2018, de https://www.gob.mx/semarnat/prensa/impulsa-semarnat-estrategianacional-para-evitar-desperdicio-de-a limentos

[2] FAO. (2017). The future of food and agriculture - Trends and challenges. Rome. Recupera do de: www.fao.org/3/a-i6583e.pdf

[3] FAO Global Perspectives Studies, using 2011 food balance sheets from FAO, 2016.

[4] Van Donselaar, K. H., van Woensel, T., Broekmeulen, R. A. C. M., \& Fransoo, J. C. (2006). Inventory control of perishables in supermarkets.

[5] Sánchez, V., \& Hasbleidy, Z. (2014). Models and configurations of supply chains in perishable goods. Ingeniería y Desarrollo, 32(1), 138154.

[6] Parfitt, J., Barthel, M., y Macnaughton, S. (2010). Food waste within food supply chains: quantification and potential for change to 2050 . 
Philosophical Transactions of the Royal Society B: Biological Sciences, 365(1554), 3065-3081.

[7] Banco Mundial. (2014). La pérdida y el desperdicio de alimentos, un obstáculo para la reducción de la pobreza. Recuperado de: http://www.bancomundial.org/es/news/press-relea se/2014/02/27/ foodloss-waste-barrier- poverty-reduction

[8] Basso, N., de Nutrición, E., \& Alimentaria, E. (2013). Las huellas del despilfarro. Alimentos argentinos. Equipo de nutrición y Educación a limentaria, 8,13 .

[9] Urquía-Fernández, N. (2014). La seguridad alimentaria en México. Salud pública de México, 56, s92-s98.

[10] Lipinski, B, Hanson, C., Lomax, J., Kitinoja, L., Waite, R., Searchinger, T., (2013). Reducing food loss and waste. Installment 2 of Creating a Sustainable Food. Future, World Resources Institute, Washington DC.

[11] Instituto Nacional de Estadística, Geografía e Informática (2018). Manejo de residuos sólidos urbanos.

[12] Murguía, E. (2018). Animal Gourmet. Recuperado de http://www.anima lgourmet.com/2015/06/09/el-camino-de-la-basura/

[13] Bustamante, C. A. G., \& Cerutti, O. M. (2016). Estado del arte de la bioenergía en México.

[14] Calderón, L. Á. F., Ocampo, E. M. T., \& Echeverri, M. G. (2012). Diseño de redes de logística inversa: una revisión del estado del arte y aplicación práctica. Ciencia e ingeniería neogranadina, 22(2), 6.

[15] Martínez, M. P., Silveira Dias, K. T., Braga Junior, S. S., \& da Silva, D. (2017). La logística inversa como herramienta para la gestión de residuos de los supermercados de venta al por menor. Revista De Gestão Ambiental E Sustentabilidade (Geas), 6(3), 150-165. doi: $10.5585 /$ geas.v6i3.519

[16] De Brito, M. P., Dekker, R., \& Flapper, S. D. P. (2005). Reverse logistics: a review of case studies. In Distribution Logistics (pp. 243281). Springer, Berlin, Heidelberg.

[17] Kim, H., Kang, J. G., y Kim, W. (2014). AN APPLICATION OF CAPACITATED VEHICLE ROUTING PROBLEM TO REVERSE LOGISTICS OF DISPOSED FOOD WASTE. International Journal of IndustrialEngineering, 21(1).

[18] Peña, I. G., Gutiérrez, A., López, C. A. y Rocha, L. B. (Octubre de 2017). CVRPTW model applied to the collection of food donations. Proceedings of the International Conference on Industrial Engineering and Operations Management, Bogotá, Colombia.

[19] Kumar, V., Kumar, V., Brady, M. y Garza-Reyes, J. A, (2017). Resolving forward-reverse logistics multi-period model using evolutionary algorithms. International Journal of Production Economics, (183), 458-469.

[20] Zhang, H., Ozturk, U. A., Zhou, D., Qiu, Y., \& Wu, Q. (2015). How to increase the recovery rate for waste cooking oil-to-biofuel conversion: A comparison of recycling modes in China and Japan. Ecological indicators, 51, 146-150.

[21] Adi, A. J., \& Noor, Z. M. (2009). Waste recycling: Utilization of coffee grounds and kitchen waste in vermicomposting. Bioresource Technology, 100(2), 1027-1030.

[22] Girotto, F., Alibardi, L., \& Cossu, R. (2015). Food waste generation and industrial uses: a review. Waste management, 45, 32-41.

[23] Zhang, H., Xu, Z., Zhou, D., \& Cao, J. (2017). Waste cooking oil-toenergy under incomplete information: Identifying policy options through an evolutionary game. Applied energy, 185, 547-555

[24] Medina, D. (2017). La comida no es basura: México, entre el hambre y el desperdicio de alimentos. Recuperado de: http://www.sinembargo.mx/28-04-2017/3202291
[25] Medina, D. (2016). ENTREVISTA | La Red PDA contra la pérdida y desperdicio de alimentos en México. Recuperado de: http://www.sinembargo.mx/23-10-2016/3106668 